\title{
Electron cyclotron resonance ion sources - physics, technology and future challenges
}

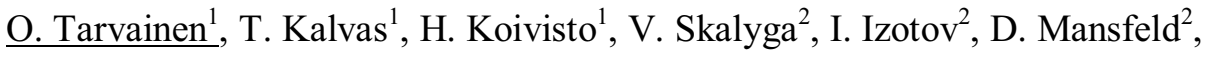 \\ ${ }^{1}$ University of Jyväskylä, Jyväskylä, Finland, olli.tarvainen@jyu.fi \\ ${ }^{2}$ Institute of Applied Physics, RAS, Nizhny Novgorod, Russian Federation
}

The performance of Electron Cyclotron Resonance Ion Sources (ECRIS), producing high charge state ions from a great variety of elements, has improved dramatically over the past decades, thus enabling significant advances in accelerator-based nuclear physics. The orderof-magnitude performance leaps of ECR ion sources, e.g. from $15 \mu \mathrm{A}$ of extracted $\mathrm{O}^{6+}$ in 1974 [1] to $4700 \mu \mathrm{A}$ in 2016 [2], result from improvements to the magnetic plasma confinement, increases in the microwave heating frequency and techniques to stabilize the plasma at high densities.

The design of modern ECR ion sources is based on semi-empirical scaling laws, suggesting most importantly that the extracted current at the peak of the ion charge state distribution scales with the microwave frequency squared [3], i.e.

$$
I_{\text {peak }} \propto \omega_{R F}^{2} .
$$

Direct comparison of $1^{\text {st }}-3^{\text {rd }}$ generation ECR ion sources operating at frequencies below $10 \mathrm{GHz}$, at 10 $18 \mathrm{GHz}$ and above $18-28 \mathrm{GHz}$, respectively, is complicated by the fact that the peak of the charge state distribution also shifts with the frequency. However, it is evident that increasing the microwave frequency improves the extracted currents of highly charge ions. Several explanations for the frequency scaling have been proposed. These include for example damping of the microwave electric field at plasma densities exceeding the critical (cut-off) value and so-called RF pitch angle scattering limiting the electron density, both scaling with the microwave frequency squared.

The magnetic field of an ECRIS is a superposition of solenoid and sextupole fields resulting to a minimum-B structure serving several purposes.

(i) The field configuration provides a closed resonance surface fulfilling the ECR-condition

$$
\omega_{R F}=\omega_{c e}=e B_{E C R} / \gamma m_{e},
$$

where $\omega_{\mathrm{ce}}$ is the electron gyrofrequency, which depends on the magnetic field strength $\mathrm{B}$ and electron energy through the relativistic $\gamma$-factor (e and $m_{e}$ are the elementary charge and electron mass, respectively). The resonant interaction with the microwave electric field assures that a sufficient number of electrons gain energies required for ionization of high charge state ions. The magnetic field fulfilling the resonance condition for non-relativistic electrons can be written conveniently as

$$
B_{E C R}[\mathrm{~T}]=f_{R F}[\mathrm{GHz}] / 28 \text {. }
$$

(ii) The minimum-B configuration results to appropriate electron confinement. The development of ECR ion sources over the past decades has led to the formulation of the following scaling laws [4] for the magnetic field:

$$
B_{i n j} / B_{E C R}=4
$$

$$
\begin{gathered}
B_{\text {rad }} / B_{E C R}=2 \\
B_{\text {ext }} \approx 0.9 B_{\text {rad }} \\
B_{\text {min }} \approx 0.4 B_{\text {rad }},
\end{gathered}
$$

where $B_{\text {inj }}, B_{\text {rad }}, B_{\text {ext }}$ and $B_{\min }$ are the magnetic field at the injection, radial wall of the plasma chamber, extraction and B-minimum, respectively.

(iii) The minimum-B configuration suppresses magnetohydrodynamic instabilities.

The scaling laws in Eqs. (1) and (4)-(7) set a practical limit for ECRIS technology relying on roomtemperature (RT) technology if the desired power consumption for generating the magnetic field is $<1 \mathrm{MW}$. In RT-ECRISs the solenoid field is created by electromagnetic coils while the radial sextupole field is formed by an array of permanent magnets. Figure 1 shows the dependence of the maximum solenoid field at the injection $\left(B_{\text {inj }}\right)$ and the sextupole field at the chamber wall fulfilling the scaling laws on the microwave frequency. Commonly applied frequencies are highlighted by the dots. The practical RT-limits of both field components, set by the power consumption and permanent magnet remanence / coer-civity, are depicted by horizontal lines crossing the data curves at $\sim 18 \mathrm{GHz}$, which is deemed as the upper frequency limit for $2^{\text {nd }}$ generation RT-ECRISs.

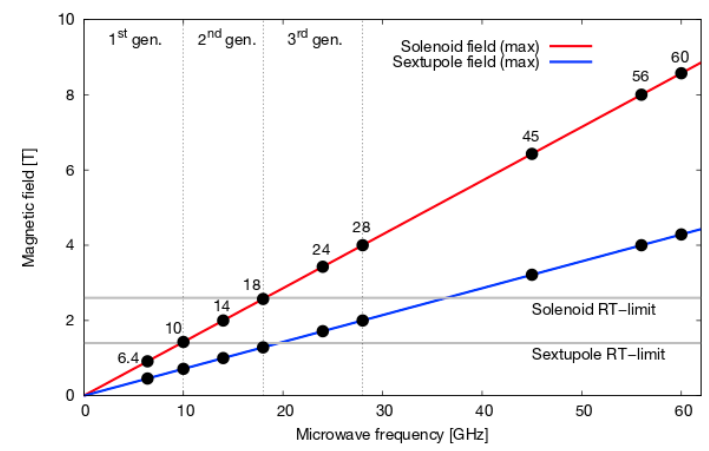

Fig. 1. Frequency scaling of the ECRIS solenoid and sextupole magnetic fields

It is evident from Fig. 1 that $3^{\text {rd }}$ generation ECRISs operating at $18-28 \mathrm{GHz}$ frequencies must rely on superconducting technologies. Building a $4^{\text {th }}$ generation ECRIS operating at microwave frequency up to $56 \mathrm{GHz}$ is feasible using state-of-the-art $\mathrm{Nb}_{3} \mathrm{Sn}$ superconducting wire [5]. Increasing the frequency beyond $56 \mathrm{GHz}$ requires either accepting a substandard field strength or R\&D on innovative ECRIS concepts, e.g. the ARCECRIS [6] feasible up to $100 \mathrm{GHz}$.

It has been recently shown [2] that the extracted currents of high charge state oxygen ion beams extracted from the $28 \mathrm{GHz}$ VENUS ECRIS increase linearly with the microwave power up to $10 \mathrm{~kW}$. Since the maximum currents scale roughly as frequency squared and the extracted current per incident watt is constant, the power 
required to operate high frequency sources up to their full capacity should also scale as frequency squared [7], i.e.

$$
P_{R F} \propto f_{R F}^{2} \text {. }
$$

The power scaling shown in Fig. 2 shows that the required power for $14,18-24$ and $28 \mathrm{GHz}$ sources is 2 $3 \mathrm{~kW}, 4-6 \mathrm{~kW}$ and $10 \mathrm{~kW}$, respectively, which matches well with the experimental evidence. This implies that operating a $4^{\text {th }}$ generation ECRIS e.g. at frequencies of 45-60 GHz would require up to $40 \mathrm{~kW}$ microwave power. Such high power poses a significant challenge on managing the localized heat load determined by the electron losses. Furthermore, the required combination of frequency / power therefore restricts the choice of applicable microwave generators as indicated in the figure.

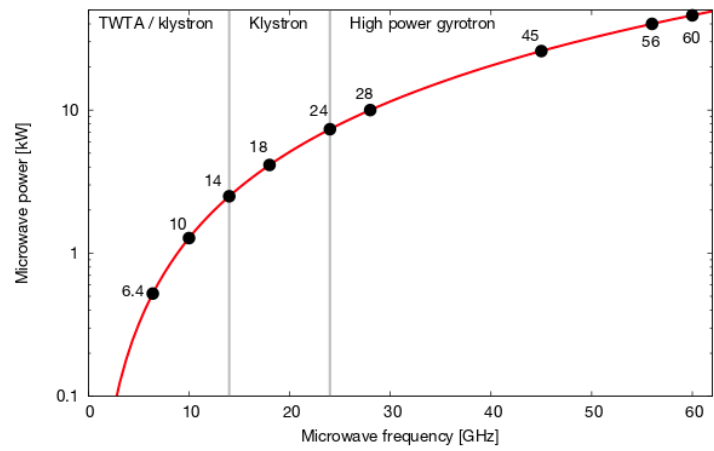

Fig. 2. Frequency scaling of the ECRIS microwave power

The ECRIS magnetic field strength at the extraction affects the ion beam quality. The emittance $\varepsilon$ of the ion beam, describing the relative position and transverse momentum of the extracted ions at each transverse plane $(x, y)$, scales (theoretically) with the magnetic field at the extraction as

$$
\varepsilon_{x, y} \propto B_{e x t} .
$$

The increase of the emittance with the magnetic field needs to be taken into account when matching the extracted beam into the acceptance of the subsequent accelerator. The presumable increase of the emittance at strong magnetic field poses yet another challenge for the R\&D of next generation ECRISs.

The magnetic field scaling laws (4)-(6) can be understood qualitatively from the point-of-view of plasma confinement and losses preferably directed towards the extraction. The scaling of the $\mathrm{B}_{\min }$ which can be written alternatively as

$$
B_{\text {min }} / B_{E C R} \approx 0.8
$$

is not evident. It has been shown [8] that when the $\mathrm{B}_{\min } / \mathrm{B}_{\mathrm{ECR}}$-ratio exceeds a certain limit, typically $0.7-$ 0.85 , the extracted currents of high charge state ions are restricted by periodic onsets of electron cyclotron instabilities. The kinetic instabilities arise from the anisotropy of the ECRIS electron velocity distribution which results from the resonant heating process affecting only the perpendicular (to B-field) velocity component of the electrons. The instabilities affect the current, longitudinal energy spread and background impurities of the extracted ion beams due to periodic loss of plasma confinement. The instability threshold can be affected by so-called two frequency heating, which allows expanding the ion source parameter space corresponding to stable plasma regime [9]. This is demonstrated in Fig. 3 showing the stabilizing effect of two frequency heating on $\mathrm{O}^{6+}$ beam current. Although, the physical mechanism explaining the suppression of the kinetic instabilities is unknown, it is evident that two frequency heating plays a crucial role in the future of ECRIS R\&D.

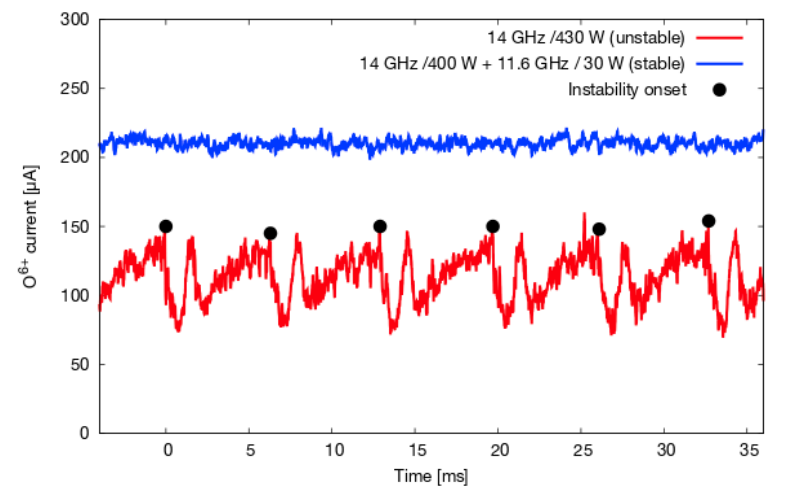

Fig. 3. Suppression of kinetic instabilities by two frequency heating. Data recorded with the JYFL 14 GHz ECRIS

It is concluded that while the performances of ECR ion sources have increased by several orders of magnitude since their conception, severe technological challenges, mainly related to the magnetic field, lie ahead if frequency scaling is pursued in the R\&D of future generation machines. Further development of techniques improving the quality and temporal stability of the extracted ion beam require deeper understanding of the underlying plasma physics.

Reported work devoted to the plasma stabilization was supported by the grant of Russian Science Foundation \# 16-12-10343.

\section{References}

1. V. Bechtold, N Chan-Tung, S. Dousson, R. Geller, B. Jacquot, and $Y$. Jongen ECR ion source for multiply-charged oxygen beams // Nucl. Instruments and Methods, 178 (1980), p. 305-308.

2. D.Z. Xie, W. Lu, J.Y. Benitez, C.M. Lyneis, and D.S. Todd, Recent Production of Intense High Charge Ion beams with VENUS // THA001 in Proc. ECRIS 2016, Busan, Korea, jacow.org

3. R. Geller ECRIS: The Electron Cyclotron Resonance Ion Sources // Annu. Rev. Nucl. Part. Sci. 40 (1990), p. 15.

4. D. Hitz, A. Girard, G. Melin, S. Gammino, G. Ciavola and L. Celona Results and interpretation of high frequency experiments at $28 \mathrm{GHz}$ in ECR ion sources, future prospects // Rev. Sci. Instrum. 73, (2002), p. 509.

5. C. Lyneis, P. Ferracin, S. Caspi, A. Hodgkinson, and G.L. Sabbi Concept for a fourth generation electron cyclotron resonance ion source // Rev. Sci. Instrum. 83, 02A301 (2012).

6. P. Suominen and F. Wenander Electron cyclotron resonance ion sources with arc-shaped coils // Rev. Sci. Instrum. 79, 02A305 (2008).

7. C.M. Lyneis Scaling Laws in Electron Cyclotron Resonance Ion Sources // MOA001 in Proc. ECRIS 2016, Busan, Korea, jacow.org

8. O. Tarvainen et al. Beam current oscillations driven by cyclotron instabilities in a minimum-B electron cyclotron resonance ion source plasma // Plasma Sources Sci. Technol. 23 (2014) 025020.

9. V. Skalyga et al. Suppression of cyclotron instabilitity in Electron Cyclotron Resonance ion sources by two frequency heating // Phys. Plasmas 22, 083509 (2015) 